\title{
DIE RÜCKLÄUFIGEN TERRASSEN AM ZÜRICHSEE UND IHRE BEZIEHUNGEN ZUR GEOLOGIE DER MOLASSE
}

\author{
NaZario Pavoni
}

Mit 3 Abbildungen

Das Zürichseetal weist vor allem in seinen mittleren Abschnitten, wo die Molasse den direkten Untergrund bildet, eine große Zahl von scharf ausgeprägten Terrassen auf, welche dem sanft geneigten Gehänge eine eigene, abwechslungsreiche Gliederung verleihen. Die Kulturlandschaft wurde dieser Morphologie weitgehend angepaßt und die Terrassen dadurch noch stärker hervorgehoben. Betrachtet man die Terrassenflächen, so erkennt man, daß sie nördlich der Linie Käpfnach - Männedorf ein Gefälle talabwärts aufweisen, während sie in der Zone südlich dieser Linie bis etwa Wädenswil - Stäfa nach SSE, das heißt talaufwärts, fallen *.

Die Frage der Entstehung der 'Terrassen am Zürichsee und insbesondere der rückläufigen Terrassen ist ein häufig diskutiertes Problem und es besteht darüber eine ansehnliche Literatur.

Es sind vor allem zwei Auffissungen, die sich seit mehr als einem halben Jahrliundert geginüberstehen, und die auch heute noch stark umstritten sind:

Alb. Heim $(1894,1919)$ und seine Schüler A. Wettstein (1885), A. Aeppli (1894) und E. GoGARTEN (1910) sprechen dem Gletscher jede größere Erosionskraft ab und vertreten die Ansicht, die 'Terrassen am Zürichsee seien Reste alter Talböden, es seien Flußerosionsterrassen (Felserosionsterrassen Alв. Hels’s). Die Rückläufigkeit der Terrassen im Gebiet von Männedorf-Stäfa einerseits und Au-Wädenswil andrerseits erklären sie mit einer flexurartigen Abbiegung dieser alten Talböden, bedingt durch ein Absinken des gesamten Alpenkörpers im Quartär. Ein erster Vorwurf gegen diese Theorie ist die Willkürlichkeit, mit der die einzelnen Terrassen zu früheren Talböden vereinigt werden. Aepplu (1894) unterscheidet mit großer Überzeugung 10 solcher Talbodensysteme, welche, eines jünger als das nächst höher gelegene, ineinander gestellt sein sollen. GoGaRTEN (1910) unterscheidet mit gleicher Überzeugung 17 Talbodensysteme. Wie deren Zahl ist auch die Lage der 'Terrassenantiklinalen und -synklinalen bei beiden Autoren erheblich verschieden (vgl. das Kärtchen in TH. ZINGG, 1934). Beide vertraten die Ansicht, daß alle Terrassen ein viel schwächeres Gefälle aufiveisen als die Molasseschichten, daß sie diese überall schief schneiden und da $\beta$ gerade damit ihre Entstehung durch Flußerosion bewiesen sei. In neuester Zeit nimmt auch M. STEIN (1948) starke Verbiegungen selbst jungquartärer Terrassensysteme im Glattal und besonders im Wasserscheidegebiet zwischen Zürichsee und oberem Glattal an.

Diesen Ansichten steht die Auffassung von E. Brückner und A. Penck (Penck und Brückner, 1909) gegenüber, die die Übertiefung des Sees der erosiven Tätigkeit des Gletschers zuschreiben. Die Terrassen, die sogenannten kleinen Terrassen BRÜCKNERS, sind nach diesen Autoren Schichtterrassen. Der See ist nach ihnen nicht durch eine quartäre Rücksenkung, sondern durch Gletschererosion und Stau durch die Endmoräne bei Zürich entstanden. Auch J. FRÜH (1919) betont in seiner Morphologie des Zürcher Oberlandes die große Bedeutung der glazialen Erosion für unser Gebiet.

Nach TH. ZINGG (1934) sind die Terrassen im Zürichseetal sogenannte Denudationsterrassen (Verwitterungs- und Abspülungsterrassen), welche sich in der Nagelfluhzone unter Umständen zu Schichtterrassen entwickeln können. In den mehr oder weniger nagelfluhfreien Gebieten seien es, im Gegensatz zur Ansicht von BRüCKNER und PENCK, keine Schichtterrassen, da die Terrassen selbst meist nicht aus den festen Sandsteinen beständen. Der Zürichsee ist nach ihm nicht durch die Rücksenkung, die auch er annimmt, bedingt. Diese habe aber im jüngern Quartär das Glattal von den Alpen abgetrennt.

Von allen Seiten wurde aber dem genauen Verlauf der Molasseschichten viel zu wenig Beachtung geschenkt. BRÜCKNER stützt sich vor allem auf den Verlauf des Kohlenlagers von Käpfnach, HEIM und seine Schüler auf ein paar Detailbeobachtungen, z. B. auf der Horgener Allmend, die ihre Ansicht unterstützen. O. HERBORDT (1907) und ZiNGG (1934) verlegen den Scheitel ihrer Antiklinale bis mehr als einen Kilometer zu weit südlich von der wirklichen Kulmination der Molasseschichten. STEIN (1948) findet sogar eine Kulmination im Risiboden, sowie „um Hombrechtikon“. Nach ZINGG beträgt die Höhendifferenz der Schichten zwischen Antiklinalscheitel und Synklinale $90 \mathrm{~m}$, welcher

* Bezeichnet man diejenigen Terrassen, welche ein Gefälle talabwärts aufiveisen, als rechtläufig, so kann man im Gegensatz dazu diejenigen, welche ein Gefälle talaufwärts aufweisen, als rückläufige Terrassen bezeichnen. 
Wert identisch sei mit dem Rücksenkungsbetrag der Terrassen nach Aepplu und Gogar'ten. In Wirklichkeit sind es aber je nach der Lage im Streichen $160-180 \mathrm{~m}$.

So hatte jede Auffassung ihre Argumente für sich, und ein neutraler Leser wußte am Ende nicht, für welche er sich entscheiden solle.

Eine erneute Überprüfung des Problems ergab sich im Zusammenhang mit der Entdeckung des „Appenzeller Granites“, eines wichtigen Kalknagelfuhleithorizontes der obern Süßwassermolasse im Gebiet des Hörnlischuttfächers, 1951 im Sihltal bei Station Sihlbrugg. Damals erhob sich die Frage, wie das Vorkommen im Sihltal zu verbinden sei mit dem bis anhin westlichsten Vorkommen des „Appenzeller Granites“ bei Feldbach am obern Zürichsee. Um diese Frage zu lösen, mußte, wie mein Lehrer, Herr Prof. Dr. R. Staub sich ausdrückte, an einem „festen Punkt“, nämlich bei Feldbach mit den Untersuchungen begonnen werden. Ich erhielt daher von ihm die Aufgabe, als Diplomarbeit an der Abteilung für Naturwissenschaften der ETH, das Gebiet zwischen Zürichsee und oberem Glattal geologisch zu bearbeiten. Dabei wurde der Verlauf der Molasseschichten im Detail wie im Großen eingehend verfolgt, und es zeigten sich bald enge Beziehungen zwischen der Morphologie des Untersuchungsgebietes und der Geologie der Molasse, die im folgenden skizziert sind.

\section{GEOLOGISCHER ÜBERBLICK}

\section{Stratigraphie und Lithologie der Molasse}

Die Profile, die wir in den vielen kleineren und größeren Tobeln des Zürichseegebietes aufnehmen können, vermitteln einen guten Einblick in die Geologie und insbesondere in die Lithologie und Stratigraphie der Molasse. Im Gebiet der rückläufigen Terrassen sind es vor allem: das Tobel bei Männedorf-Auf Dorf, das Tobel, welches vom Allberg herunterkommt, das Risitobel, das Tobel bei Strick-Grundhalden, das Schleifetobel, das Dorlentobel und die Tobel, die bei Feldbach in den See münden, und auf der linken Seeseite das Aabachtobel, das bei Käpfnach mündet, das Meilibachtobel und die Tobel oberhalb Wädenswil.

Nagelfluh, Sandsteine, Mergel und Kalke sind die Gesteinsarten, aus welchen sich die Molasse zusammensetzt. Der Anteil der Mergel schwankt je nach Profil zwischen 25 und 50\%, der Anteil der Sandsteine zwischen 20-40\%, derjenige der Nagelfluhen zwischen weniger als $10 \%$ und mehr als $45 \%$. Die Kalke, die stets als Knollenkalke mit Beimengungen von detritischem Material auftreten, beteiligen sich mit durchschnittlich 6,5\% am Molasseprofil. Bei den Sandsteinen ist zwischen Knauersandsteinen, welche in typischer Ausbildung mergelfrei sind, und Mergelsandsteinen zu unterscheiden. Knauersandsteine und Nagelfluhen treten rinnenförmig auf, d. h. es sind Ablagerungen in früheren, tertiären Flußbetten. Da es sich bei beiden um ganz entsprechende Bildungen handelt, lassen sich sehr häufig seitliche und vertikale Übergänge von Knauersandstein in Nagelfluh beobachten. Ein Knauersaudstein kann immer ein Aequivalent einer Nagelfluhbank sein und umgekehrt. Diese Tatsache ist von großer Bedeutung bei der Verfolgung von Nagelfluhen als Leithorizonte und auch für die Morphologie des Gebietes. Von NW nach SE nimmt der Anteil der Nagelfluhen am Gesamtprofil der Molasse auf Kosten der Mergel und Sandsteine zu; denn wir nähern uns in dieser Richtung dem Zentrum des Hörnlischuttfächers, wo die Nagelfluhschüttungen mehr als $95 \%$ ausmachen können (H. TANNER, 1944).

\section{Tektonik der Molasse}

Die Tektonik der Molasse läßt sich nur mit Hilfe von Leithorizonten genau feststellen. Direkte Messungen von Streichen und Fallen sind äußerst unsicher und of irreführend, da viele ungünstige Faktoren mitspielen. Auch handelt es sich bei den Fallwerten meist um sehr geringe Beträge. Neben vielen Nagelfluh-Knauersandsteinbänken wurden vor allem zwei Leithorizonte verfolgt:

1. Das Niveau des "Appenzeller Granites". Es wird charakterisiert durch eine lithologisch auffällige Kalknagelfluh, durch einen ausgezeichneten hellgrauen Mergelkalk und durch einen feinen hellgrauen Sandstein. Bis anhin von Abtwil (St. Gallen) bis nach Feldbach am Zürichsee bekannt, konnte ich das Niveau am rechten Seeufer von Oetikon, wo es wieder aus dem See auftaucht, über Männedorf-Auf Dorf, Uetikon, Meilen bis nach Herrliberg verfolgen, wo es ein zweites Mal unter dem Seenniveau verschwindet. Zudem fand es sich in großer Ausdehnung im obern Glattal.

2. Der "Wetterkalk von Hombrechtikon ". Es ist ein oft mehr als zwei Meter mächtiger, rötlicher Knollenkalk, der eine Ausdehnung von mehr als $100 \mathrm{~km}^{2}$ besitzt.

Aus dem Verlauf der einzelnen Nagelfluhbänke und den beiden erwähnten Leithorizonten ergaben sich übereinstimmend folgende Strukturen der Molasse im Gebiet der rückläufigen Terrassen der rechten Seeseite und der Glattalschwelle: Eine flache, asymmetrische Antiklinale zieht, auf das Niveau des "Appenzeller Granites" bezogen, von Männedorf-Auf Dorf über Weidenbad, Hohrüti, Holzhausen, Büchlen nach Itzikon und sticht nördlich von Punkt 512,9 in den Hügel bei Birch. Nach Herbordt (1907) geht eine Antiklinale bei Tobelholz-Herrgass, also $1,5 \mathrm{~km}$ südöstlich unseres Antiklinalscheitels durch. Auch Tн. ZiNGg (1934) legt seine Antiklinale hier durch. Eine solche hier durchzulegen ist sicher falsch. Gerade im Tobelholz konnte eine gute Fallmessung und bei Punkt 558, anläßlich der Straßenverbreiterung, zwei weitere solche mit übereinstimmenden Werten gemacht werden. Alle drei Messungen ergaben ein Schichtfallen von 5,5-6 $6^{\circ}$ nach SSE. Zudem befinden wir 
uns hier in einer ausgesprochenen Schichtrippenlandschaft, M. STEIN (1948, Seite 44) findet sogar eine Antiklinale im Risiboden, sowie „um Hombrechtikon“; dabei ist gerade der Risiboden eines der eindruckvollsten Beispiele für eine prachtvolle - wie ja der Name sagt - ebene, schwach SSE fallende Schichtfläche, welche gebildet wird durch den Wetterkalk von Hombrechtikon. Daß die Antiklinale bei Grüningen-Itzikon durchzieht, wird am schönsten durch das Wiederauftauchen des „Appenzeller Granites " in diesem Gebiet belegt. Man kann sie darum mit Recht Grüninger-Antiklinale nennen. Sie ist sicher identisch mit der Antiklinale von Käpfnach auf der linken Zürichseeseite (vgl. E. LETSCH [1899] und A. von Moos [1946]).

Während der weite Nordschenkel der Grüninger-Antiklinale sehr flach, maximal mit $2,5-3^{\circ}$, absinkt, zeigen die Schichten im kürzeren Südschenkel größere Fallwerte. Am stärksten mit etwa $6-7^{\circ}$, fallen sie in der Gegend von Uelikon-Risiboden-Kuhweid (Schichtrippenlandschaft)-Herrenholz-Herrgass-Adletshausen. Der Lützelsee liegt mit seiner Längsachse genau im Streichen der Molasseschichten. Im Gebiet von Redlikon-Hombrechtikon-Homberg nimmt das Fallen ab, und zugleich ist auch die Streichrichtung nicht mehr so konstant ENE-WSW.

Bei Kehlhof/Stäfa-Ranghausen-Eichwies-Berlikon-Wiedenswil haben wir die flache Synklinalmulde erreicht. Deutlich kommt auf einer Strukturkurvenkarte zum Ausdruck, wie sich bei EichwiesTobel die Synklinale zu einem eigentlichen Becken ausweitet und vertieft. Dieses Becken ist der Grund, warum sich gerade hier mehrere Bäche, vergleichbar den Hauptnerven eines Ahornblattes, in die Landschaft eingeschnitten haben, sich bei Punkt 433 sammeln und gemeinsam die schwach NNW fallenden Schichten durchbrechen, um bei Feldbach in den Zürichsee zu münden. Auch diese Synklinale findet ihre westliche Fortsetzung auf der linken Zürichseeseite in der Synklinale von Wädenswil.

In der Gegend von Wiederzell-Neuhaus-Schlatt-Uerikon beginnen die Schichten wieder nach Norden zu fallen. Das NNW-Fallen nimmt nach SSE ständig zu. Am Herrenhölzli maß ich ein solches von $3,5-4^{0}$. Bei Hüllistein sind es $6-7^{\circ}$ und bei Jona nach Th. ZINGG (1934) bereits $25^{\circ}$.

\section{MORPHOLOGIE}

\section{Die morphologischen Faktoren}

Das Gebiet der rückläufigen Terrassen auf beiden Seiten des Zürichsees und ebenso die Schwelle vom Zürichseetal ins obere Glattal sind gänzlich frei von glazialen Ablagerungen. Es ist die Molasse, die sozusagen überall den direkten Untergrund bildet.

Die Gesteine der Molasse lassen sich in Bezug auf ihre morphologische Wirksamkeit in zwei Hauptgruppen einteilen:

a) Die Nagelfuhen und Knauersandsteine. Diese Gesteine der Rinnenfazies treten wegen ihrer größeren relativen Härte gegenüber den mergelhaltigen Gesteinen der Molasse und wegen ihrer oft beträchtlichen Mächtigkeit in der Landschaft als Rippen, Hügel oder Schichtflächen, welche begrenzt sind von Steilstufen, hervor.

b) Die mergelhaltigen Gestein. Wegen ihrer geringen Härte bilden sie in der Landschaft den Untergrund von Mulden oder die basale Partie von Steilstufen mit ausflachendem Gehänge als Übergang zur nächst tiefergelegenen Steilstufe. Die Gebiete, wo Mergelgesteine die Unterlage bilden, sind oft versumpft (Quellenaustritte aus hangenden Sandstein- oder Nagelfluhbänken) und verrutscht und aus diesen Gründen Aufschlüsse selten.

Die Kalke nehmen eine besondere Stellung ein. Sie sind wohl hart, jedoch meistens von zu geringer Mächtigkeit, um im Landschaftsbild wirksam zu sein. Wo sie aber größere Mächtigkeit aufweisen, treten sie morphologisch sehr stark in Erscheinung, als ausgedehnte Schichtflächen. Das weitaus beste Beispiel dafür ist der «Wetterkalk von Hombrechtikon »: In der flachen Synklinallandschaft von Redlikon - Hombrechtikon - Wolfhausen - Egelsee bildet er auf einer Fläche von mehr als fünf Quadratkilometern den direkten Untergrund; das ist mehr als die Hälfte der gesamten Oberfläche dieses Gebietes. Die weiten Schichtflächen dieses Kalkes, auf denen die Nagelfluhhügel wie zurückgelassene Kuchen liegen, bestimmen den Charakter dieser Landschaft.

Neben der Gesteinsart ist die tektonische Lagerung der Schichten von großer Wichtigkeit für die Morphologie; denn bei gleicher Gesteinszusammensetzung und gleichen Denudationsfaktoren können ganz verschiedene Landschaftsformen entste- 
hen je nach dem, ob die Schichten horizontal gelagert sind, oder ob sie ein gewisses Fallen aufweisen. Ferner ist der Grad der W'echsellagcrungsintensität, das heißt des Wechsels von harten und weichen Schichten, eines Gesteinsverbandes, von bestimmendem Einfluß auf die Gestaltung der Oberflächenformen.

Während die umliegenden Gebiete meist eine starke Bedeckung durch glaziale und fluvioglaziale Ablagerungen aufweisen, ist die breite Schwellenzone zwischen Zürichsee und oberem Glattal, mit welcher das Gebiet der rückläufigen Terrassen von Männedorf bis Stäfa in enger Beziehung steht, und ebenso das Gebiet der rückläufigen Terrassen auf der linken Seeseite fast gänzlich frei von eiszeitlichen Ablagerungen.

Unsere Untersuchungen, die sich.eingehend mit dem Verlauf der Molasseschichten befaßten, führten überall im Untersuchungsgebiet immer zum gleichen Ergebnis :

Die Neigung der Terrassenflächen, welche wir wohl in einem etwas engeren Sinne auffassen als Aeppla (1894), Gogakten. (1910), Zingg (1934) und Stein (1948) - welche Autoren den Begriff Terrasse in großzügigerer Weise gebrauchen - ist stets identisch mit dem Fallen der Molasseschichten an der betreffenden Stelle.

Die Unterlage der Terrassen wird immer gebildet durch eine relativ mächtige, harte Schicht, meist durch Nagelfluh- oder Knauersandsteinbänke, die heute allerdings zum Teil schon ziemlich stark verwittert sind, oder durch Kalke. Die Terrassen sind Schichtterrassen. Diese Tatsache wird durch unzählige Beispiele auch in nagelfluhfreien Zonen, so im Gebiet von Meilen, Herrliberg, Erlenbach bewiesen. Natürlich kann man Ausnahmen finden, die sich aber bei genauer Untersuchung meist zwanglos erklären lassen (siehe unten, Seite 221), ohne dabei irgendwelche Gegenargumente zu liefern. Wegen solcher weniger, möglicher Ausnahmen aber die so vielfältig und eindeutig belegte Tatsache, daß es sich bei den Terrassen am obern Zürichsee um Schichtterrassen handelt, übersehen oder ablehnen zu wollen, wäre falsch. Umgekehrt ist es allerdings nicht gesagt, daß jede harte, mächtige Bank eine Schichtterrasse bilden muß.

Fluviatile Erosion kann diese ausgedehnten Schichtflächen nicht freigelegt haben. Sie vermochte bis heute die Morphologie, wie sie der Würmgletscher nach seinem Rücken zurückließ, keineswegs zu verwischen; sie hat die Terrassenränder erst in einigen Tobeln angenagt. Es ist allerdings möglich, daß die Anlage mancher Tobel auf Gletscherbäche zurückzuführen ist und daß diese Schmelzwasser die Terrassenränder zum Teil noch schärfer herauspräpariert haben. Auf jeden Fall aber handelt es sich dabei nur um sekundäre Bildungen.

Die Verwitterung, auf die ZingG (1934) allem Anschein nach die Entstehung seiner Denudationsterrassen zurückführt, arbeitet in unsern Regionen wegen der starken Vegetationsbedeckung nicht so schnell, um in der relativ kurzen Postglazialzeit so weite Schichtflächen freilegen zu können, die zudem frei sind von Schutt. Es ist zum Beispiel ganz unerklärlich, wie in der flachen Synklinallandschaft von Redlikon - Hombrechtikon - Egelsee die sehr ausgedehnten Schichtflächen auf dem Wetterkalk durch Verwitterung entstehen konnten. Wir sollten in einem solchen Fall zum mindesten große Schuttmassen finden, welche sich bei der Verwitterung der über dem Kalk liegenden Nagelfluhen gebildet hätten. Wir finden aber nirgends Anzeichen von solchen, hingegen an einigen wenigen Stellen, so in der Mulde von Neuhaus südlich Wolfhausen, Reste von glazialen Ablagerungen direkt auf dem Kalk. Es besteht auch keine Möglichkeit, daß diese Schuttmassen durch fließendes Wasser (z. B. Abspülung, Schichtfluten) hätten wegtransportiert werden können; denn das ganze Gebiet ist sehr flach, immer etwa auf Kote 500 m, z. T. abflußlos oder unterirdisch entwässert. Ein flächenmäßiges «Abwaschen》des Verwitterungs- 
schuttes würde zudem durch die sehr starke Verkarstung der Kalkoberfläche verunmöglicht, da der Niederschlag an Ort und Stelle versickert.

Der Schluß, daß die Schichtterrassen durch selektive glaziale Erosion herausgearbeitet wurden, ist daher aus vielen Gründen der gegebene. Damit ist auch das oben erwähnte Fehlen glazialer Akkumulationsformen im Gebiet der rückläufigen Terrassen und der Glattalschwelle erklärt: Es ist ein Gebiet mit glazialer Erosion. Warum die Gletscher gerade in diesem Gebiet besonders intensive Erosionstätigkeit entfalteten, ist damit zu erklären, daß sie, zum mindesten der Würmgletscher, mit ihrer Unterlage vom tiefer gelegenen Zürichseetal über die Schwelle zum Glattal hinaufsteigen mußten. Die Verengung des Zürichseetales beim Eintritt in die Klus durch die Grüninger-Antiklinale ist ein weiterer Grund für die glaziale Erosion im Gebiet der Terrassen.

Es ist erstaunlich, auf wie weite Strecken der Gletscher dort, wo es ihm in seinen Lauf paßte, stets derselben Schicht, zum Beispiel dem Wetterkalk, folgte und alle Deckschichten immer bis gerade auf diese Schicht wegerodierte, um sich so eine möglichst glatte Unterfläche und Gleitfläche zu schaffen.

Der Gletscher konnte nicht stets der gleichen Schichtfläche folgen, sonst hätte er zum Beispiel dem «Appenzeller Granit » entlang von Kote $360 \mathrm{~m}$ in der Synklinale bis Kote $540 \mathrm{~m}$ auf der Antiklinale steigen müssen. Er folgte der Schicht nur so lange, als deren Verlauf mit seinem Lauf mehr oder weniger zusammenfiel, und in diesem Fall tat er es mit Vorliebe. Wo die Abweichung des Schichtverlaufes vom allgemeinen Gletscherverlauf zu groß wurde, und wo die Schichtfläche, welcher der Gletscher eben noch gerne gefolgt war, zu weit über oder unter den allgemeinen Verlauf der Gletscherunterfläche reichte, verließ er die Schicht und folgte je nach dem einer stratigraphisch tiefer oder höher gelegenen Bank. An den Stellen, wo ein solcher Übergang stattfindet, treffen wir nie Terrassen.

Es kommt häufig vor, daß eine Nagelfluh- oder Knauersandsteinrinne auskeilt. Dies bedeutet dann auch das Ende einer Terrasse. Im Gebiet von Meilen, Herrliberg und Erlenbach trifft man viele kleinere Terrassen an, die ziemlich unvermittelt aus dem Gehänge hervortreten und markante Punkte bilden. Der ihnen vorgelagerte, gegen den See schauende Steilhang trägt auch heute noch fast immer einen Rebberg. Oben gegen den Terrassenrand hin wird der $\mathrm{Hang}$ meist so steil, $\mathrm{da} \beta$ auch die Reben nicht mehr gehalten werden können und das nackte Gestein als Felswand zu Tage tritt. Dann erkennen wir immer, daß es eine lokale Knauersandsteinbank ist, die die Terrasse bildet.

Neben der Eigenschaft der glazialen Erosion, selektiv zu arbeiten - welche Tatsache uns gerade im Gebiet der rückläufigen Terrassen auf Schritt und Tritt eindrücklich entgegentritt - ist es noch eine weitere Eigenart, die sie scharf unterscheidet von der Flußerosion, und welche sich einem beim Studium der Gehänge beidseits des Zürichsees aufdrängt. Die Gletschererosion arbeitet vor allem in die Breite, die Wirkung des Eises wird also besonders stark sein auf die Talseiten. Während das Wasser fast unbekümmert um das Gestein nach der Tiefe und nach einer Normalgefällskurve strebt und die Gehänge des Tales zum Nachrutschen zivingt, indem es sie ihrer Grundlagen beraubt, und so indirekt das Tal verbreitert, kann der Gletscher die Talseiten aktiv und selektiv in beliebiger Höhenlage bis dort, wo er hinaufreicht, bearbeiten. Wird in flach gelagerten Molasseschichten durch fluviatile Erosion ein $\mathrm{Tal}$ geschaffen, so zeigt dieses im Prinzip V-Form. Fließt später ein Gletscher in dieses $\mathrm{Tal}$, so arbeitet er selektiv in die Breite. Nach seinem Rückzug wird das Tal viel breiter sein und Schichtterrassen aufweisen: Entstehung der Terrassen am obern Zürichsee.

Die Terrassen am obern Zürichsee

Die Besprechung der morphologischen Faktoren ergab, daß dort, wo der Verlauf der Molasseschichten mehr oder weniger übereinstimmt mit dem allgemeinen 


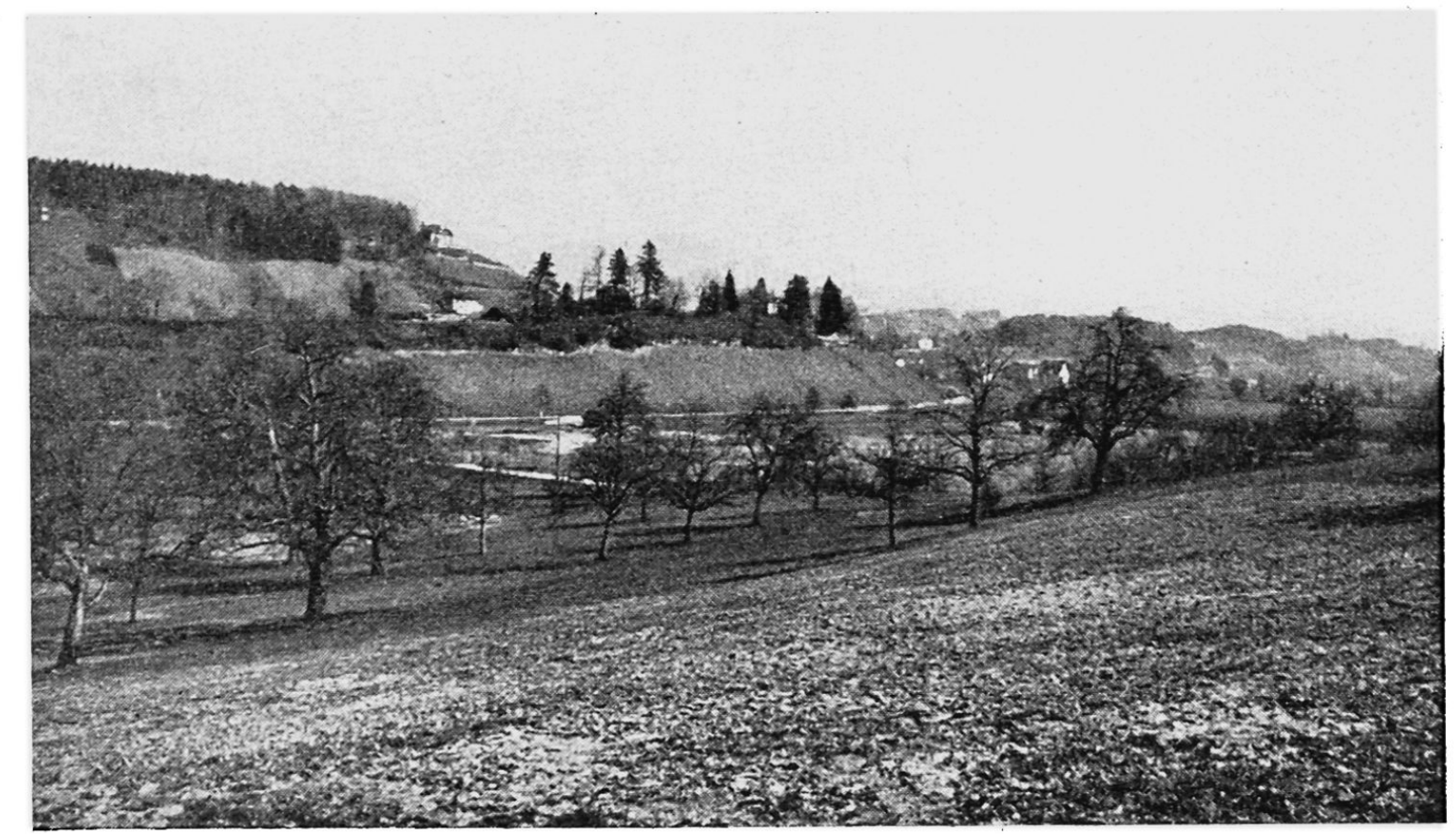

Abb. 1 Nagelfluhschichtterrassen bei Feldbach. Vordergrund: Schirmensee. Mittelgrund: Östliches Ende der zwei km langen Schichtterrasse, auf der die Bahnlinie von Uerikon nach Feldbach verläuft.

Der Verfasser verdankt diese Foto der Freundlichkeit von Herrn F. Pestalozzi, Herrliberg.

Verlauf der Gletscherunterfläche, das fließende Eis ausgedehnte Schichtterrassen herausmodelliert, daß aber dort, wo dies nicht der Fall ist, der Gletscher sich nicht stark um den Schichtverlauf kümmert, es vielleicht zur Ausbildung einer Schichtrippenlandschaft mit gerundeten Formen kommt und bei allzugroßen Abweichungen das Eis sich gänzlich unbekümmert um hart und weich seinen Weg bahnt.

Die Voraussetzungen für die Ausbildung von Schichtterrassen waren wegen der verschiedenen Strukturen der Molasse auf beiden Seiten des Zürichsees nicht die gleichen. Sie waren viel günstiger, fast ideal, auf der rechten Seeseite. Die Terrassen der rechten Zürichseeseite seien aus diesem Grunde auch ausführlicher behandelt.

Die Terrassen zwischen Feldbach und Stäfa. Den schönsten Eindruck vom treppenartig gestuften Gehänge zwischen Feldbach und Stäfa erhalten wir, wenn wir vom linken Ufer, etwa von Wädenswil - Richterswil aus, über den See blicken. Wir sehen, wie die ausgeprägte Schichtrippenlandschaft bei Jona in ganz allmählichem Übergang über Hüllistein, Lenggis zum Gebiet der nur noch leicht nach NNW fallenden Schichtterrassen bei Schlatt, Palm, Eggrüti östlich von Feldbach wird. Wie große Treppenstufen liegen sie vor der flachen Schwelle, die zum obern Glattal führt. Längs der Straße von Feldbach nach Uerikon folgen wir in Streichrichtung einer zwei Kilometer langen Terrasse (Abb. 1). Sie wird immer gebildet durch dieselbe Nagelfluhbank, welche entlang dem Terrassenrand durchgehend aufgeschlossen ist. Die Eisenbahnlinie zwischen Uerikon und Feldbach verläuft zu einem großen Teil auf ihr. Östlich von Uerikon oder selbst vom fahrenden Zug aus erkennen wir deutlich, daß die Terrasse mit der Nagelfluhschicht leicht nach NW einfällt. Sie liegt ungefähr $45 \mathrm{~m}$ über dem Niveau des «Appenzeller Granites ». Über dieser großen Terrasse kann man östlich von Uerikon noch weitere solche feststellen, z. B. bei Pkt. 462,7. Auch hier liegt nur eine geringe Rasendecke über der NNW fallenden Nagelfluhbank.

Zwischen Uerikon und Stäfa finden wir eine prachtvolle Terrasse über der andern, so bei Sternen, bei Eich-Laubisrüti und Rütihof. Immer bestätigt es sich aufs neue, daß es Schichtterrassen sind; denn entlang jedem Rand finden wir eine $\mathrm{Na}$ - 


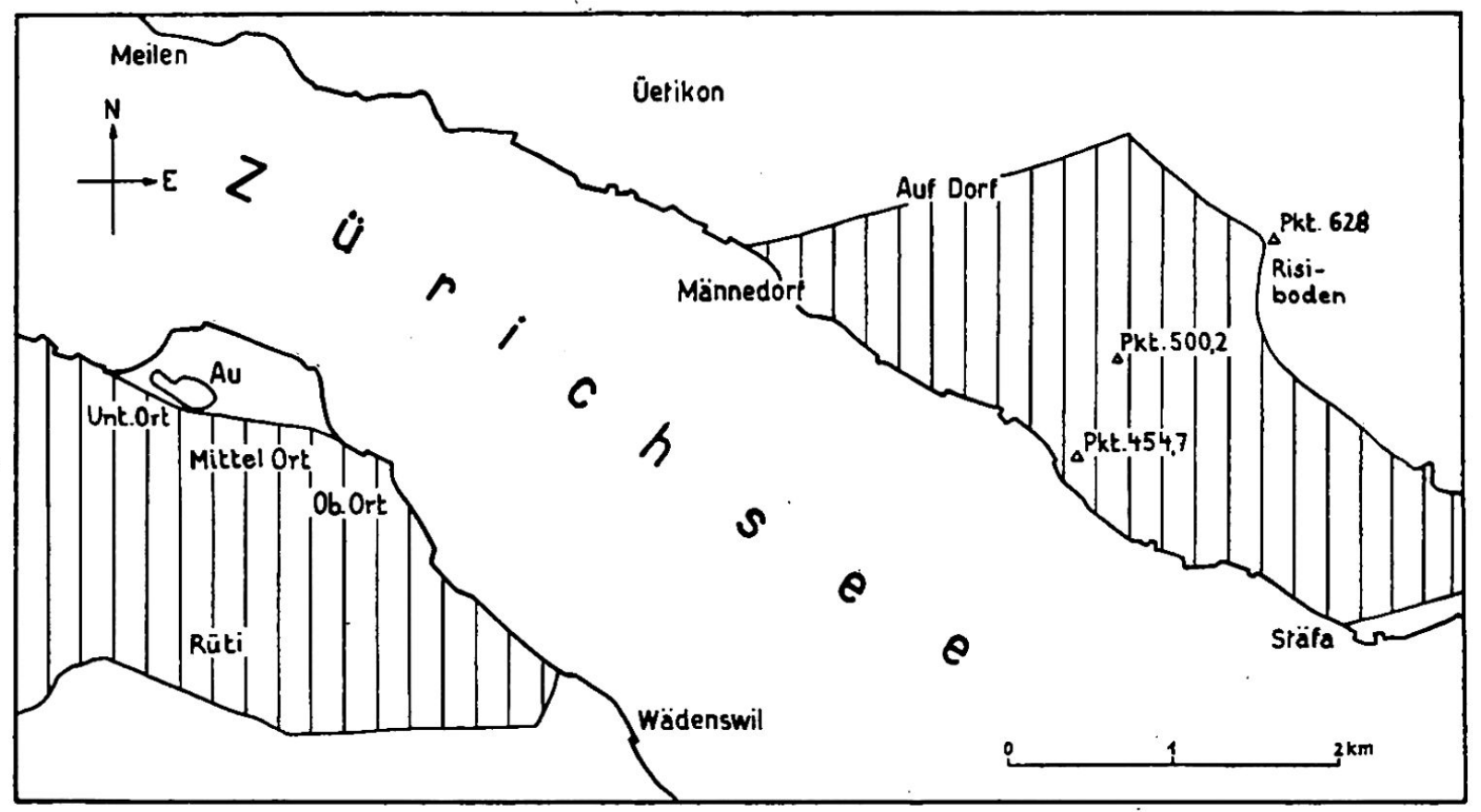

Abb. 2 Übersichtsskizze. Senkrecht schraffiert das Gebiet der rückläufigen Terrassen

gelfluhbank aufgeschlossen. Aber nicht jede Nagelfluhschicht bildet unbedingt eine Terrasse. So sind zum Beispiel im Hang östlich Bauertacker - Grundhalden, in welchen das Risitobel eingeschnitten ist, mehrere Nagelfluhbänke enthalten. Gegen Norden wird die Terrassenlandschaft zwischen Stäfa und Feldbach durch eine letzte Steilstufe bei Kote 490-500 m von der ganz anders gearteten, flachen Synklinallandschaft von Redlikon - Hombrechtikon - Wolfhausen - Egelsee abgegrenzt. Wenn man vom Zürichsee her, etwa von Stäfa oder Feldbach kommend, in wechselndem Anstieg die Terrassen und die ihnen vorgelagerten, rebenbestandenen Steilhänge durchquert hat, ist man immer wieder von neuem überrascht, wie unvermittelt sich nach diesem letzten steilen Rand die weite Synklinallandschaft vor einem öffnet.

Die Klus von Männedorf. Nordwestlich von Stäfa beginnen die Schichten gegen die Grüninger-Antiklinale anzusteigen. Uelikon, Binz, Grundhalden liegen alle auf Schichtflächen. Von Binz nach Bauertacker ist die Straße immer auf derselben Schichtfläche. Bei Kniebreche überwindet sie eine Steilstufe, und von dort bis zur Paßhöhe verläuft sie wieder auf einer nächst höhern Schichtfläche (Abb. 3).

Die Terrassen auf der linken Seeseite. Die Voraussetzungen für die Entstehung von ausgedehnten Schichtterrassen waren, wie wir schon gesehen haben, auf der rechten Talseite günstiger als auf der linken, darum nämlich, weil das Zürichseetal die Synklinale von Wädenswil - Stäfa und die Käpfnach-Grüninger-Antiklinale schief durchbricht. Das linksufrige Gelände am obern Zürichsee weist zudem eine starke Bedeckung durch Moränen und Schotter auf. Das Gebiet der rückläufigen Terrassen aber ist auch auf dieser Seite frei von glazialen Ablagerungen. Die Molasse bildet direkt den Untergrund.

In vollständiger Übereinstimmung mit den Beobachtungen auf der rechten Seeseite zeigt es sich, daß auch die rückläufigen Terrassen der linken Zürichseeseite Schichtterrassen sind. Es seien nur erwähnt die Knauersandsteinterrassen bei Unter Ort - Mittler Ort, ferner die Schichtrippen bei Dächelwies, Mittler Rüti, Winterberg und Hangenmoos. Die größte und eindrücklichste Schichtfläche ist diejenige von Mittler Rüti, Vorder Rüti, Bühlenebnet. Sie zeigt starkes SSE Fallen (ca. 10\%). Entlang dem sehr scharf ausgeprägten Terrassenrand, welcher von Pkt. 573,0 über Pkt. 567, Pkt. 553,3 nach Pkt. 544 zieht, ist überall die darunter- 


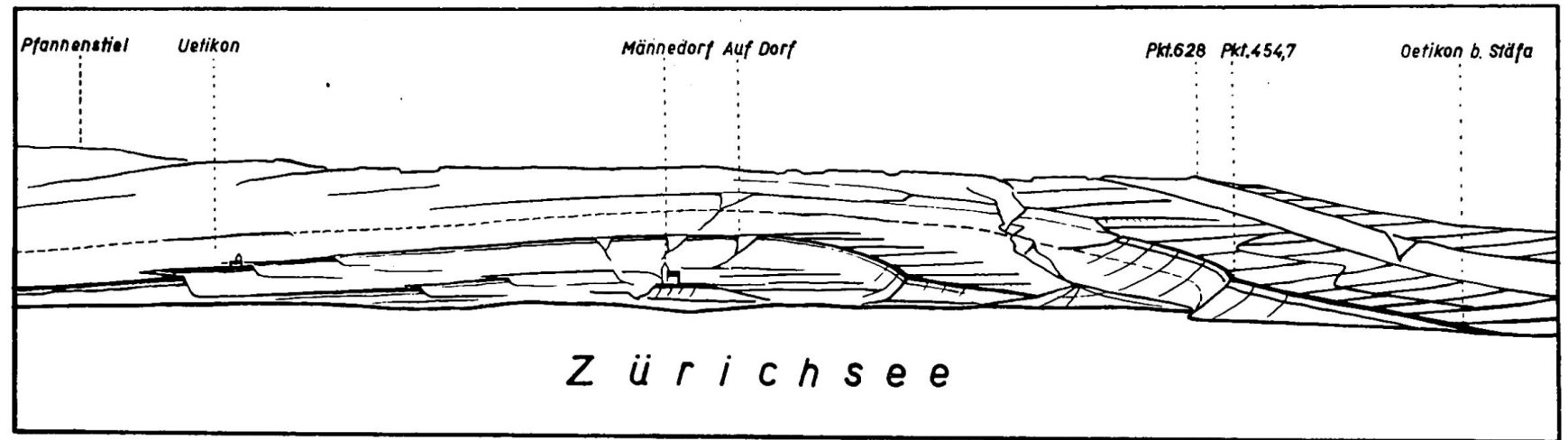

Abb. 3 Die Klus von Männedorf

(Gesehen vom linken Seeufer. Vereinfachte schematisierte Darstellung)

Gestrichelt: das Niveau des „Appenzeller Granites.“ Dem Zürichsee entlang ist der Anstieg der Schichten eindrucksvoll zu erkennen in der mächtigen Nagelfluhbank, die vom Stäfnerstein her kommend bei Oetikon aus dem See auftaucht und, durchbohrt vom Tunnel der Eisenbahn, in NW Richtung zu Pkt. 454,7 hinaufsteigt. Fast in einem rechten Winkel zieht sie von hier NNE zu Pkt. 500,2, quert als Knauersandstein bei Kote $540 \mathrm{~m}$ das Tobel und erreicht unter Pkt 563,7 ihre Kulmination. Bei Oetikon-Hotwil, unter Pkt 454,7 bilden die Molasseschichten, vor Abtragung geschützt durch die eben erwähnte Nagelfuh, eine "Nase“, einen Vorsprung in den See hinaus, der sich als morphologisch sehr bedeutsame Linie auch unterseeisch verfolgen läßt (siehe unten). Im Westen" dieses Vorsprunges liegt die Ebene von Außerfeld-Mutzmalen, welche, vergleichbar einem mächtigen Amphitheater, von dem prächtigen Rebgelände des Latterberges umrahmt wird. WETTsteIn (1885, Seite 54) und AEprLI (1894) deuten sie als große Serpentine. AEpPLI schreibt in seiner Arbeit über „Erosionsterrassen und Glazialschotter in ilırer Beziehung zur Entstehung des Zürichsees“ auf Seite 22: „Daß hier (bei Mutzmalen) die Sache etwas schwieriger zu überblicken ist, liegt hauptsächlich darin, daß hier eine prachtvolle, alte Serpentine den westlichen Teil der untern Terrassen herausgeschnitten hat. Dadurch ist der Steilhang der Buchhalde bei Außerfeld (Männedorf) entstanden " Nach unserer Auffassung ist die Morphologie in diesem Gebiet einfacher zu erklären: Das Ziirichseetal durchbricht zwischen Stäfa und Meilen einerseits und Wädenswil und Horgen andererseits, als ursprünglich diesem Gebiet einfacher zu erklären: Das Zïrichseetal durchbricht zwischen Stäfa und Meilen einerseits und Wädenswil und Horgen andererseits, als ursprünglich
fluviatil angelegtes und durch die Gletscher verbreitertes Tal, die flache Molasseantiklinale wnd bildet hicr eine Klus. Durch die vorhin erwähnte, mächtige Nagelfluhbank der Buchhalde entstand am südöstlichen Eingang in die Klus eine „Einengung“, die allerdings durch die Gletscher kräftig verbreitert wurde und heute noch in Vorsprung bei Pkt. 454,7 und dessen unterseeischer, morphologischer Fortsetzung erhalten ist. Sie trennt auf der Linie OetikonWädenswil das nordwestliche, tiefe Seebecken vom süd-östlichen, untiefen und flachgründigen Teil des Sees (vergl. R. STAUB, 1938).

Bei Auf Dorf-Männedorf zieht der Scheitel der Käpfnach-Grüninger-Antiklinale durch, wir befinden uns hier im zentralen Teil der Klus. Auf Dorf liegt selbst auf einer der schönsten und ausgedehntesten Schichtterrassen des ganzen Zürichseetales. Im flachen Nordschenkel der Antiklinale gelegen, senkt sie sich von Auf Dorf (Kote $490 \mathrm{~m}$ ) in nordwestlicher Richtung über Allmend, Uetikon-Kleindorf, Kirchbühl, Dollikon bis gegen Feldmeilen, wo sie schon bald wieder das Niveau des Sees erreicht hat. Immer ist es dieselbe mächtige Nagelfluh-Knauersandstein-Bank, ivelche auf einer Länge von mehr als vier Kilometern die Unterlage dieser Terrasse bildet. Sie bildet zugleich den nördlichen Abschnitt der Klus. 
liegende Molassenagelfuh aufgeschlossen. Dort wo die Molasseschichten die Synklinale erreicht haben, finden wir auch keine rückläufigen Terrassen mehr.

\section{Das Tal}

Das Zürichseetal ist ursprünglich durch Flußerosion entstanden. Es wurde später durch die Gletscher kräftig verbreitert und übertieft. In seinem Verlauf ist es durch die Strukturen der Molasse bestimmt: Von Rapperswil bis Stäfa - Richterswil liegt es im Streichen der Molasseschichten von Stäfa bis Meilen und von Richterswil bis Horgen ist es ein Quertal schief durch die Synklinale von WädenswilStäfa und durch die Käpfnach-Grüninger-Antiklinale (Klus von Männedorf). Der untere Teil des Zürichseetales ist durch Bruchlinien tektonisch vorgezeichnet.

Es gab einst ein mächtiges Ringen der Gewässer um die erste, entscheidende Durchbruchstelle durch die Käpfnach-Grüninger-Antiklinale, mußte doch später das Haupttal hierdurch zu liegen kommen. Dieser Durchbruch erfolgte an der geologisch prädestinierten Stelle zwischen Käpfnach und Männedorf. Der große Preis, um den es ging in diesem Wettkampf: Er liegt vor uns in seiner einzigartigen Schönheit, es ist das Zürichseetal.

\section{ZUSAMINENFASSUNG}

Im Bereich der rückläufigen Terrassen am ohern Zürichsee fehlen, in auffälligem Gegensatz zu den Gebieten der weitern Umgebung, glaziale Akkumulationsformen fast gänzlich. Die Molasse bildet sozusagen überall den direkten Untergrund. Es handelt sich um ein Gebiet mit glazialer Erosion. Die Terrassen sind Śchichtterrassen (PENCK \& BRÜCKNER [1909]). Diese wurden durch die selektive Erosion der Gletscher herausgearbeitet. Nagelfluh- und Knauersandsteinbänke bilden jeweils die Unterlage der Terrassen. Es sind also sicher nicht Reste von alten Talböden (Wettstein [1885], Heim [1894, 1919], Aeppli [1894], Gogarten [1910] Stein [1948]) und auch keine Verwitterungs- und Abspülungsterrassen (Denudationsterrassen von ZINGg [1934]). Die fluviatile Erosion hat die Morphologie, wie sie der Würmgletscher zurückließ, bis heute nur in ganz geringem Maße anzutasten vermocht. Die rückläufigen Terrassen liegen alle im Südschenkel der Käpfnach-Grüninger-Antiklinale. Wir finden nirgends Anhaltspunkte, aus denen wir schließen könnten, daß im Quartär irgendeine Rücksenkung stattgefunden hätte. Der Verlauf der Molasseschichten, welcher eingehend verfolgt wurde, zeigt, daß es im Zürichseegebiet weder eine Verbiegung jungquartärer Terrassensysteme im Sinne von Stein, noch eine postglaziale Abtrennung des Glattales von den Alpen durch Rücksenkung und Verstärkung der Amplitude der Antiklinale und Synklinale, wie ZINGG dies postuliert, noch eine alpine Randflexur im Sinne Herm's und seiner Schüler gibt. Das Zürichseetal ist in seiner Lage und in seinem Verlauf ursprünglich durch die Strukturen der Molasse bestimmt.

\section{LITERATURVERZEICHNIS}

Aeppli, A. (1894): Erosionsterrassen und Glazialschotter in ihrer Beziehung zur Entstehung des Zürichsees. Diss. Univ. Zürich. - FRÜH, J. (1919): Zur Morphologie des Zürcher Oberlandes. Vierteljahrsschrift N. G. Zürich, 64. Jg. - GoGarten, E. (1910): Über alpine Randseen und Erosionsterrassen, im besonderen des Linthtales. Diss. Univ. Zürich. - Heim, Alb.: (1894): Die Entstehung der alpinen Rand-Seen. Geologische Nachlese Nr. 1. Vierteljahrsschrift N. G. Zürich, 39. Jg. - HEIM, Alb. (1919): Geologie der Schweiz, Bd. I, Leipzig. - HerbordT, O. (1907): Geologische Aufnahme der Umgegend von Rapperswil-Pfäffikon am Zürichsee. Diss. Univ. Zürch. - LETsCH, E. (1899): Die schweizerischen Molassekohlen östlich der Reuß, Beitr. z. geol. Karte d. Schweiz, Geotechn. Serie, Lfg. 1. - Moos, A. von (1946): Die Kohlebohrungen bei Sihlbrugg und die Molassestrukturen um Zürich. Ecl. geol. Helv., Bd. 39. - PavoNI, N. (1952): Molassestudien zwischen östlichem Pfannenstiel und der Jona. Diplomarbeit an der Abt. X, ETH. Manuskript. - PENCK, A. und BrúCKNER, E. (1909): Die Alpen im Eiszeitalter. Bd. II, Leipzig. - Staub, R. (1938): Prinzipielles zur Ent- 
stehung der alpinen Randseen. Ecl. geol. Helv. Bd. 31. - Stein, M. (1948) : Morphologie des Glattales. Diss. Univ. Zürich. - TANNER, H. (1944): Beitrag zur Geologie der Molasse zwischen Ricken und Hörnli. Mitt. d. Thurg. N.G., Heft 33. - Wetrstein, A. (1885): Geologie von Zürich und Umgebung. Diss. Univ. Zürich. - Z INGG, TH. (1934): Erläuterungen zu Atlasblatt 7 (Mönchaltorf, Hinwil, Wädenswil, Rapperswil) d. geol. Atlas d. Schweiz, mit Karte.

\section{LES TERRASSES A PENTE INVERSE DES BORDS DU LAC DE ZURICH ET LEURS RELATIONS AVEC LA MOLASSE}

L'article traite de la nature des terrasses à pente inverse signalées au bord du lac de Zurich. Contrairement aux régions environnantes, les dépôts glaciaires y manquent à peu près totalement. Le sol est formé par la molasse, roche typique du Miocène supérieur. L'érosion glaciaire y a sculpté les formes. Les terrasses sont donc des „banquettes", formées par les couches dures (poudingues et grès) de la molasse découvertes par l'action sélective des glaciers. Ce ne sont donc pas des restes de fonds de vallées antérieures. Nous n'avons trouvé aucune preuve d'un affaissement des Alpes pendant le Quartenaire, évènement qui, selon Heim, serait la cause de la pente inverse des terrasses. Celles-ci sont situées sur le flanc Sud de l'anticlinal molassique de Käpfnach-Grüningen.

\section{TERRAZZI RETROGRADI DEL LAGO DI ZURIGO E LA LORO RELAZIONE CON LA GEOLOGIA DELLA MOLASSA}

Si discute la natura dei terrazzi cosiddetti retrogradi presenti nella vallata del Lago di Zurigo. In contrasto con le regioni circostanti, vi mancano in modo quasi completo i depositi glaciali. La molassa, tipica roccia del miocene superiore, vi forma direttamente il sottosuolo. E una regione di erosione glaciale. I terrazzi risultano di strati duri della molassa (Nagelfluh e arenarie) rimasti meno intaccati dall'azione erosiva selettiva del ghiacciaio : non si tratta dunque di fondi di valli anteriori. Non si è infatti trovato nessun indizio che stesse a dimostrare un abbassamento delle Alpi durante il quaternario, fenomeno che secondo Heim dovrebbe essere considerato come causa della retrocessione dei terrazzi. I terrazzi in parola sono situati sul fianco sud dell'anticlinale molassica di Käpfnach-Grüningen.

\section{DIE ZUSCHÜTTUNG DES THUNERSEES}

\section{At.Fred Steiner}

Als Teil des oberen Aaretales ist das Seebecken des Thunersees, wie alle Formen der Erde, in steter Umbildung begriffen. Ein Vorgang dieser Umbildung, nämlich die Zuschüttung, findet in dieser Arbeit besondere Beachtung.

\section{DIE NEUE THUNERSEEKARTE 1:25000}

Meine Arbeit stützt sich auf die neue Thunerseekarte 1:25000 der eidg. Landestopographie, die nicht veröffentlicht wird, aber als Grundlage für die Landeskarte 1:50000 dient. Die bisherigen Kartendarstellungen des Thunersees geben nur in großen Zügen die Form der Seewanne wieder, ohne jegliche Einzelheiten des Reliefs. Einzig die großen Deltas sind darin zu erkennen. Umso mehr Beachtung verdient deshalb die Karte, die durch Herrn A. CharLes, stellvertretender Leiter des techn. Dienstes der Landestopographie in Bern in den Jahren 1946 bis 1949 neu gelotet und völlig neu gezeichnet wurde. Die bisherigen Karten wurden auf Grund von 408 Lotungen total oder 8 pro $\mathrm{km}^{2}$ gezeichnet, wobei die Anordnung der Lotungen meistens querschnittartig erfolgte. Völlig anders ist Herr CharLes vorgegangen, indem er, sobald sich eine Besonderheit des Reliefs zeigte, in nächster Umgebung davon viele Lotungen durchführte, total 2155 oder 45 pro $\mathrm{km}^{2}$. Dadurch ist eine Karte mit einem großen Reichtum an Einzelformen geschaffen worden.

\section{DAS GESAMTVOLUMEN DES SEES}

Um mir über die vertikale Gliederung einer glazialen, der Zuschüttung ausgesetzten Seewanne genauere Kenntnis zu verschaffen, berechnete ich die Isobathenflächen von $345 \mathrm{~m}$ ü. M. bis zur Oberfläche des Sees in $558 \mathrm{~m}$. Anhand dieser horizontalen Flächenabschnitte (siehe $T a b .1$ ) habe ich in der heutigen Seewanne eine Dreiteilung in der Vertikalen vorgenommen, die ich als 1. Deltazone; 2. Mittelzone; 3. Bodenzone bezeichne. 